\title{
Microleakage Comparative Evaluation of RMGIC and Alkasite with and without Adhesive System in Class V Cavity: An In Vitro Study
}

\author{
Dennis Dennis ${ }^{1}$, Sondang Pintauli ${ }^{2}$, Shinta Debora ${ }^{3}$
}

\begin{abstract}
Aim and objective: This study aimed to compare microleakage of resin-modified glass ionomer cement (RMGIC) and alkasite with and without adhesive system in class $V$ cavity.

Materials and methods: Thirty class $V$ cavities were prepared on the buccal surface. All prepared samples were randomly divided into three groups. Group I was restored with RMGIC, group II was restored with Cention N after the application of adhesive system, and group III was restored with alkasite. Samples were subjected to 200 thermocycles between temperature baths at $5{ }^{\circ} \mathrm{C}$ and $55^{\circ} \mathrm{C}$. Samples were stained with $2 \%$ methylene blue. The teeth were sectioned longitudinally, and the sections were then observed under stereomicroscope at a magnification of 20x. Results: Statistical analysis showed that there was less microleakage in Cention $\mathrm{N}$ using adhesive system in both occlusal margin and gingival margin.

Conclusion: There were significant differences between RMGIC, alkasite with adhesive system, and alkasite without adhesive system in class $\mathrm{V}$ cavity. Least microleakage at the occlusal margin and gingival margin was seen with Cention $\mathrm{N}$ using adhesive system and highest microleakage at the RMGIC.

Clinical significance: Cention $\mathrm{N}$ using the adhesive system affects the microleakage in the class $\mathrm{V}$ restoration. The study theorizes that the material restorations showed less microleakage in the occlusal margin than in the gingival margin.

Keywords: Adhesive, Alkasite, Class V restoration, Microleakage, RMGIC.

The Journal of Contemporary Dental Practice (2021): 10.5005/jp-journals-10024-3130
\end{abstract}

\section{INTRODUCTION}

Class V lesions are those occurring at the cervical aspect of the buccal or lingual surfaces of teeth. Conventionally, based on the etiology, a class $\mathrm{V}$ lesion is broadly classified as carious and non-carious lesions. ${ }^{1}$ Carious class $V$ cervical lesions are the result of chronic plaque accumulation at the cervical third of the tooth in conjunction with a significant intake of fermentable carbohydrates. An additional risk factor is a reduction in salivary flow and salivary quality due to xerostomia. ${ }^{2}$

Non-carious cervical lesions develop as a result of normal and abnormal or pathological wear with a variable morphology ranging from shallow grooves to broad dished-out lesions to large notched or wedge-shaped defects. Such lesions include abrasion, erosion, and abfraction; these lesions at the cemento-enamel junction can affect the tooth sensitivity, plaque retention, caries incidence, structural integrity, and pulp vitality; they present unique challenges for successful restoration. ${ }^{3}$

Class $\mathrm{V}$ restorations also present a challenging and technically demanding situation, because the gingival margin, usually located in dentin or cement, is considered a critical factor in governing the marginal adaptation. One of difficulties in class $V$ restoration is eliminating or reducing gap formation on the gingival wall. Several restorative techniques have been proposed to minimize shrinkage due to polymerization and to also achieve better marginal adaptation in class $\mathrm{V}$ cavities. Bond strength to enamel is usually greater than bond strength to dentin. ${ }^{4}$

Another challenge is tooth isolation. Intrinsic anatomical and morphological characteristics of the cervical region create
${ }^{1,3}$ Department of Conservative Dentistry, Faculty of Dentistry, Universitas Sumatera Utara, Medan, Sumatera Utara, Indonesia

${ }^{2}$ Department of Preventive and Public Health Dentistry, Universitas Sumatera Utara, Medan, Sumatera Utara, Indonesia

Corresponding Author: Dennis Dennis, Department of Conservative Dentistry, Faculty of Dentistry, Universitas Sumatera Utara, Medan, Sumatera Utara, Indonesia, Phone: +628126555068 , e-mail: dennis @usu.ac.id

How to cite this article: Dennis D, Pintauli S, Debora S. Microleakage Comparative Evaluation of RMGIC and Alkasite with and without Adhesive System in Class V Cavity: An In Vitro Study. J Contemp Dent Pract 2021;22(7):735-738.

Source of support: Nil

Conflict of interest: None

limitations in placement of the rubber dam and clamp. Proper isolation is very difficult, sometimes impossible, when lesions extend proximally or under the gingiva. Sometimes, part of the structure cannot be isolated and the dam promotes restorative material accumulation and excess. ${ }^{4}$

One of the major requisites for the longevity of a restoration is its ability to adapt to the cavity walls, the failure of which would lead to microleakage. Microleakage is the clinically undetectable passage of bacteria, fluids, molecules, and ions between the cavity wall and the restorative materials applied to it. Microleakage can lead to staining around the margins of restoration, postoperative sensitivity, secondary caries, restoration failure, pulpal pathology 
or pulpal death, and partial or total loss of restoration. Controlling microleakage has always been an important goal of operative dentistry. Various restorative materials have been tried and tested for the same, with each having its own advantages and disadvantages. ${ }^{5}$

Resin-modified glass ionomer was introduced in the late 1980 s. $^{6}$ It release fluoride and bond chemically to tooth structure. ${ }^{7}$ Resin-modified glass ionomer was introduced as an attempt to overcome the problems such as moisture sensitivity and low physical properties associated with the conventional GIC. ${ }^{8}$

Cention N is an "alkasite" restorative. Alkasite refers to a new category of filling material, which like compomer or ormocer materials is essentially a subgroup of the composite class. ${ }^{5}$ It is a tooth-colored, dual-cured restorative material available in powder and liquid form. It contains various alkaline ion releasing fillers like fluoride, calcium, and hydroxyl in the powder. These alkaline ions neutralize acidic ions surrounding the restoration. Monomer from liquid enhances the flowability of the material and adapts to the smear layer. ${ }^{9}$

Alkasite also includes special patented filler (isofiller) that acts as shrinkage stress reliever, and due to its low elastic modulus, this shrinkage stress reliever within alkasite reduces polymerization shrinkage and microleakage. Alkasite offers a cost-effective substitute for amalgam and also fulfills the need for an esthetic bulk fill material in the posterior region. ${ }^{10}$

Therefore, the aim of the current study is to compare the microleakage level of the resin-modified glass ionomer cement (RMGIC) restorative material, alkasite restorative material with adhesive system, and alkasite restorative material without adhesive system in class $\mathrm{V}$ restoration.

\section{Materials and Methods}

This type of research is experimental in vitro laboratory with post-test study without control group design. The study sample consisted of 30 human permanent maxillary premolars.

Thirty human permanent maxillary premolars teeth extracted for orthodontic reasons were selected. Selection criteria for teeth were absence of caries, restoration, and lack of evidence of cracks. All samples were cleaned and stored in saline. A standardized class $V$ cavities were prepared on the buccal surface of each tooth. The cavity preparation was standardized to $2 \mathrm{~mm}$ of depth, $4 \mathrm{~mm}$ mesio-distal width, and $2 \mathrm{~mm}$ height in occluso-gingival direction. Teeth were randomly divided into three groups of ten cavities each $(n=10)$.

1. Group 1: Dentin conditioner was applied on the cavity for 20 seconds, then rinsed off; then RMGIC (GC Fuji II LC, GC America) was placed and light-cured for 20 seconds.

2. Group 2: Universal adhesive (Tetric N-Bond Universal, Ivoclar Vivadent) was applied on the cavity and light-cured for 20 seconds; then, alkasite(Cention $N$, Ivoclar Vivadent) was placed in the cavity with bulk technique and light-cured for 20 seconds.

3. Group 3: Alkasite (Cention N, Ivoclar Vivadent) was placed in the cavity with bulk technique and light-cured for 20 seconds.

After restoration, teeth were stored in saline at $37^{\circ}$ for 24 hours. Samples were then subjected to $200 \times$ thermocycles between temperature at $5^{\circ}$ and $55^{\circ}$. Cycles in each bath lasted for 30 seconds with a transfer time of 10 seconds. The root apices were sealed with utility wax, and the entire tooth surface was coated with two layers of commercial nail varnish to within $1.0 \mathrm{~mm}$ of the restoration.
The samples were immersed in methylene blue $2 \%$ for 24 hours at $37^{\circ} \mathrm{C}$ and then washed under water and dried. The teeth were sectioned longitudinally through the center of the restorations with disk bur. The dye penetration of the occlusal and gingival margins of each section was evaluated independently by two evaluators using $20 \times$ stereomicroscope to evaluate the degree of microleakage around the restorations.

Scoring system used was similar to that used by Meshram. Value and its inferences used in the present study are as follows: ${ }^{9}$

1. Score 0: no evidence of microleakage

2. Score 1: dye penetration up to half of cavity depth

3. Score 2: microleakage more than half of the depth of cavity wall

4. Score 3: dye leakage involves axial wall

\section{Statistical Analysis}

The data were collected, tabulated, and statistically analyzed using Kruskal-Wallis test and Mann-Whitney test to evaluate differences among the experimental groups at a significant level. $p<0.05$ was taken to be statistically significant.

\section{Results}

Data were analyzed using Kruskal-Wallis test and Mann-Whitney test (Tables 1 and 2). Microleakage values for all groups were lesser at occlusal margin as compared to those at the gingival margin interface. Differences in occlusal margin microleakage ( $p=0.003)$ and gingival margin microleakage $(p=0.06)$ were significant between group 1, group 2, and group 3 (Table 1). In occlusal margin, the mean percentage microleakage of RMGIC was found to be 16.75 , while that of alkasite with adhesive system was 8.65 and that of alkasite without adhesive system was 21.10 . In the gingival margin, the mean percentage microleakage of RMGIC was found to be 17.00 , while that of alkasite with adhesive system was 10.00 and that of alkasite without adhesive system was 19.50 (Table 1).

Pairwise comparison was done using Mann-Whitney test (Table 2). There were significant $(p<0.05)$ differences in microleakage at the occlusal margin between groups 1 and $2(p=0.016)$, and groups 2 and 3 ( $p=0.002)$. Further, there were no significant $(p>0.05)$ differences between groups 1 and $3(p=0.108)$ (Table 2). Differences in microleakage at the gingival margin were significant between groups 1 and $2(p=0.032)$, and groups 2 and $3(p=0.005)$, and there were no significant differences between groups 1 and $3(p=0.146)$. Samples in group 2 showed the least leakage at the occlusal margin and gingival margin when compared to other groups (Table 2).

Table 1 shows that mean microleakage score of group 1 is higher than that of group 2 and lower than that of group 3.

Table 1: Result of Kruskal-Wallis test

\begin{tabular}{lllll}
\hline \multicolumn{1}{c}{ Group } & $N$ & Mean & $p$ \\
\hline \multirow{2}{*}{ Occlusal margin } & 10 & 16.75 & \\
& $\begin{array}{l}\text { Alkasite with } \\
\text { adhesive system } \\
\text { Alkasite without } \\
\text { adhesive system } \\
\text { RMGIC }\end{array}$ & 10 & 8.65 & 0.003 \\
& $\begin{array}{l}\text { Alkasite with } \\
\text { adhesive system } \\
\text { Alkasite without } \\
\text { adhesive system }\end{array}$ & 10 & 21.10 & \\
& 10 & 10.00 & 0.006 \\
\hline
\end{tabular}


Table 2 : Result of Mann-Whitney test

\begin{tabular}{lcc}
\hline & \multicolumn{2}{c}{ Score microleakage } \\
\cline { 2 - 3 } Group & Occlusal margin & Gingival margin \\
\hline I and II & 0.016 & 0.032 \\
I and III & 0.108 & 0.146 \\
II and III & 0.002 & 0.005 \\
\hline
\end{tabular}

The mean microleakage score of group 2 is lowest compared to the other groups. The mean microleakage score of group 3 is highest compared to the other groups. Microleakage values for all groups were greater at the occlusal margin compared to the gingival margin.

Group of RMGIC under 20× magnification of stereomicroscope demonstrated that the occlusal and gingival margins reveal dye penetration up to half of cavity depth and involve axial wall (Fig. 1). Group of alkasite with adhesive application under $20 x$ magnification of stereomicroscope demonstrated that some specimens showed dye penetration up to almost half of cavity depth on the occlusal and gingival margins but some specimens showed no microleakage on the occlusal and gingival margins (Fig. 2). Group of alkasite without adhesive application under $20 \times$ magnification of stereomicroscope showed dye leakage involving full cavity depth and axial wall on the occlusal and gingival margins (Fig. 3).

\section{Discussion}

One of the prime factors for the success of a restoration is the marginal adaptability of the restorative material. Adaptability of Cention N and RMGIC (GC Fuji II LC, GC America) in class V cavities is checked in this study. Clinically, cervical lesions extend to different tooth substrates like enamel at the occlusal and dentin at the gingival margins. ${ }^{10}$

Microleakage is the clinically undetectable passage of bacteria, fluids, molecules, or ions in microgaps $(10-6 \mu \mathrm{m})$ between a cavity wall and the restorative material applied to it. Microleakage is still a concern in restorative dentistry, as it has been related to pulp alterations, sensitivity, and secondary caries, which are the most common causes of restoration failure. ${ }^{11}$
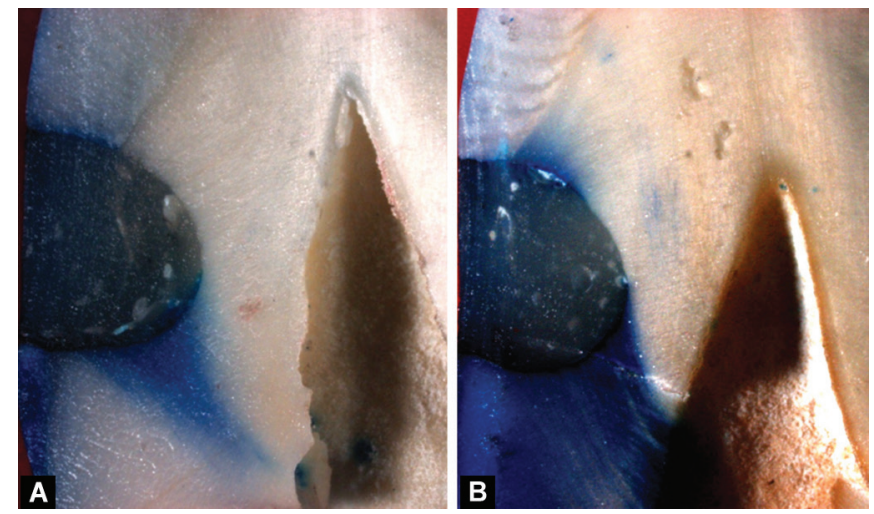

Figs $1 \mathrm{~A}$ and $\mathrm{B}$ : Group of resin-modified glass ionomer cement under $20 \times$ magnification of stereomicroscope. (A) Occlusal margin reveals dye penetration up to half of cavity depth, while gingival margin reveals dye leakage involving axial wall; (B) Occlusal margin and gingival margin reveal dye leakage involving axial wall

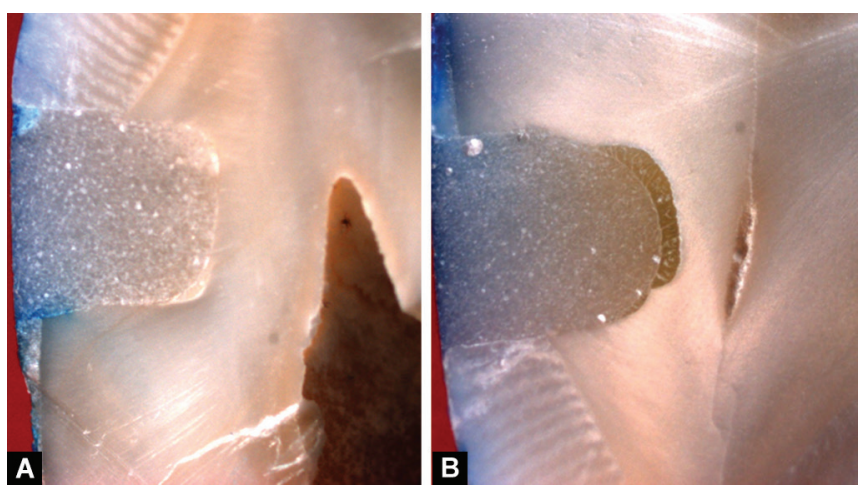

Figs $2 A$ and B: Group of alkasite with adhesive application under $20 \times$ magnification of stereomicroscope. (A) Occlusal margin and gingival margin reveal dye penetration up to almost half of cavity depth; (B) Occlusal margin and gingival margin reveal no microleakage

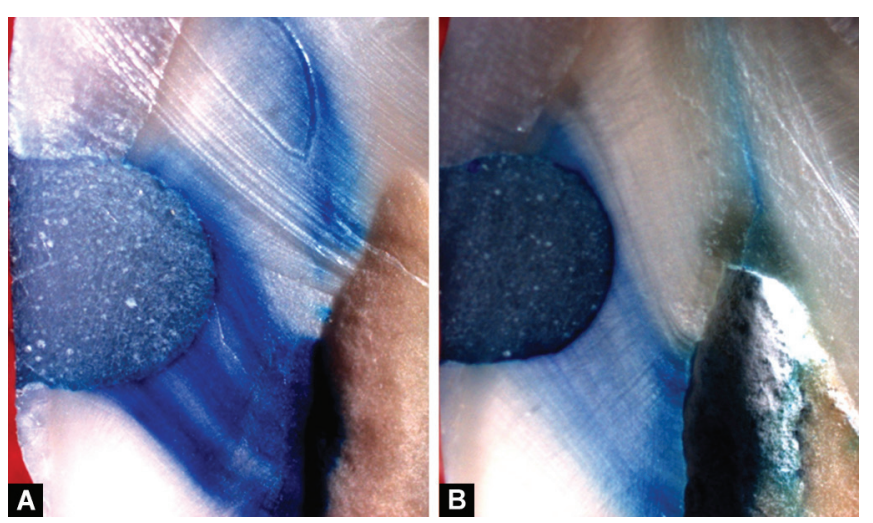

Figs 3 A and B: Group of alkasite without adhesive application under $20 \times$ magnification of stereomicroscope. Occlusal margin and gingival margin reveal dye leakage involving axial wall

RMGIC (GC Fuji II LC, GC America) consists of powder and liquid. The liquid component usually contains a water solution of polyacrylic acid, HEMA, and polyacrylic acid modified with methacrylate. The powder component contains fluoroaluminosilicate glass particles of a conventional GIC plus initiators, such as camphorquinone (CQ), for light curing and/or chemical curing. The acid-base reaction begins upon mixing and continues after polymerization at a much slower rate than for conventional GICs because less water is present and the reaction is much slower in the solid phase than in the liquid phase. ${ }^{12}$

Advantages of RMGIC are extended working time, control on setting, good adaptation, chemical adhesion to enamel and dentin, fluoride release, improved aesthetics, and low interfacial shrinkage stress. Disadvantages of RMGIC are shrinkage on setting and limited depth of cure especially with more opaque lining cements. ${ }^{13}$

Restoration with RMGIC (GCFuji II LC, GC America) showed more microleakage than Cention $\mathrm{N}$ with adhesive system but less than Cention $\mathrm{N}$ without adhesive system. Samanta et al. designed a study to compare and evaluate the microleakage in class V cavity filled with flowable composite resin, glass ionomer cement, and alkasite. According to the results, flowable composite exhibited the highest microleakage followed by glass ionomer, and the least microleakage was shown by alkasite. ${ }^{10}$ 
Restorations with alkasite using adhesive system demonstrated the least microleakage at the occlusal margin and gingival margin than RMGIC and Cention N without adhesive system. At the occlusal margin, it showed the least microleakage than the gingival margin within each restoration. Our findings are similar to Meshram et al. who found less microleakage at the enamel restoration interface. ${ }^{14}$

The liquid of alkasite (Cention N, Ivoclar Vivadent) comprises dimethacrylates and initiators, while the powder contains various glass fillers, initiators, and pigments. Due to the presence of crosslinking methacrylate monomers in combination with a stable, efficient self-cure initiator, alkasite (Cention N, Ivoclar Vivadent) exhibits a high polymer network density and better degree of polymerization over the complete depth of the restoration. ${ }^{5}$

Minimizing shrinkage stress is particularly important in a full-volume replacement material that is applied in bulk. Cention, therefore, contains a shrinkage stress reliever with a low modulus of elasticity. It acts like a microscope spring, attenuating the forces generated during shrinkage. Reduced polymerization shrinkage should translate as lower volumetric shrinkage, improved marginal integrity, and reduced shrinkage stress force over the restorative surface/on the adhesive bond. ${ }^{11}$

Cavities restored with alkasite using adhesive showed less leakage between restoration and tooth structure than those restored without alkasite because the interface is largely sealed as an acid-resistant and resin-dentin inter diffusion zone, i.e., hybrid layer. Our findings are similar to that of the study conducted by Lopes and Burgess who found when alkasite applied without adhesive, demonstrated an acceptable gap between dentin and the restorative material of about $20 \mu \mathrm{m}$ in average. The smear layer that occluded the tubules plays an important role as a barrier to prevent the fluid movement inside the tubules. ${ }^{14,15}$ Meanwhile, cavities restored with alkasite using the adhesive system showed that the interface between alkasite and dentin was largely sealed as so-called an acid-resistant and resin-dentin inter diffusion zone. ${ }^{15}$ Use of dentin adhesive system improves marginal seal of restorative material and tooth interface. They have proven to be effective at reducing but not eliminating microleakage. ${ }^{14}$

The limitation of this study was that all samples were extracted teeth that might have some alteration from vital teeth that still have moisture and collagen fiber. Therefore, future clinical study should be performed in order to compare with current study.

\section{ConcLusion}

Within the limitation of this study, our findings were significantly different between RMGIC (GC Fuji II LC, GC America), alkasite (Cention $N$, Ivoclar Vivadent) with adhesive system, and alkasite (Cention $N$, Ivoclar Vivadent) without adhesive system in class $\mathrm{V}$ cavity. Restoration with RMGIC shows more microleakage than alkasite using adhesive system and alkasite without adhesive system. The clinical significance of this study is that alkasite restorative material combined with the application of adhesive has better adhesion than alkasite without adhesive and RMGIC in class $V$ restoration.

\section{Authors' Contributions}

DD, SP, and SD conceived the original idea and designed the experiment. DD and SD performed the experiment, and collected and analyzed data. DD, SP, and SD contributed to the interpretation of the results. DD wrote the manuscript with support from SP and SD. All authors read and approved the final manuscript.

\section{Availability of Data and Materials}

The data analyzed during this present study are available from corresponding author on reasonable request.

\section{Consent to Publish}

The authors have given the consent for publication.

\section{References}

1. Suma B, Savitha S, Nandini S, et al. Management of class V lesions based on etiology. J Conserv Dent 2007;10(4):141-147. DOI: 10.4103/0972-0707.43038.

2. Hempton TJ, Ovadia R, McManama JC, et al. Addressing cervical class V lesions. J Profession Excellence Dimens Dent Hyg 2010;8(3):48-51.

3. Patil TN, Saraf PA, Penukonda R. Non-carious cervical lesions: an update. J Indian Dent Assoc2017;11(5):32-39.

4. Perez CR. Alternative technique for class $V$ resin composite restorations with minimum finishing/polishing procedures. Oper Dent 2010;35(3):375-379. DOI: 10.2341/09-310-TR.

5. Kaur M, Khanna R, Malhotra S, et al. Stereomicroscopic evaluation of microleakage using cention $\mathrm{n}$ and conventional glass ionomer cement. Indian J Compr Dent Care 2018;8(2):1143-1145.

6. Sakaguchi RL, Powers JM. Craig's restorative dental materials. 13th ed. Philadelphia: Elsevier; 2012. p. 184.

7. Berzin DW, Abey MC, Wilkie CA, et al. Resin-modified glass-ionomer setting reaction competition. J Dent Res 2010;89(1):82-86. DOI: 10.1177/0022034509355919.

8. Almuhaiza M. Glass ionomer cement in restorative dentistry. J Contemp Dent Pract 2006;17(4):331-336. DOI: 10.5005/ jp-journals-10024-1850.

9. Meshram PV, Meshram VS. Comparative evaluation of microleakage around class $\vee$ cavities restored with new alkasite material and two different flowable composite resin. Int J Curr Res 2018;10(4): 67780-67782. DOI: 10.4103/ijdr.IJDR_767_17.

10. Mann JS, Sharma S, Maurya S, et al. Cention n: a review. Int J Curr Res 2018;10(5).

11. Mazumsdar P, Das A, Das UK. Comparative evaluation of microleakage of three different direct restorative materials (silver amalgam, gic, cention $\mathrm{n}$ ) in class II restorations using stereomicroscope. Indian J Dent Res 2019;30(2):277-281.DOI: 10.4103/ijdr.IJDR_481_17.

12. Anusavice, Shen, Rawls. Philip's science of dental materials. 12th ed. Elsevier; 2012. p. 325-327.

13. Garg N, Garg A. Textbook of operative dentistry. New Delhi: Jaypee Brothers Medical Publishers; 2010. p. 402-404.

14. Meshram P, Meshram V, Palve D, et al. Comparative evaluation of microleakage around class $v$ cavities restored with alkasite restorative material with and without bonding agent and flowable composite resin. Indian J Dent Res 2019;30(3):403-407.DOI: 10.4103/ijdr. IJDR_767_17.

15. Scientific documentation: cention $\mathrm{n}$ ivoclarvivadent $A G$ research \& development scientific service. 2016. 